\title{
Analysis of indexes CPO-D and IHOS in patients with Diabetes Mellitus of Tulancingo Hidalgo, Mexico
}

\author{
Pedro Macbani Olvera ${ }^{1}$, Juan Alberto Moctezuma Alviter ${ }^{2}$, \\ Dariana Lyly Hernández Fernández ${ }^{2}$, Martínez Javier², \\ Leyva Santillán Emmnuel ${ }^{2}$ Patricia Vázquez Alvarado ${ }^{3}$, \\ Mariela Lizbeth Cobián Diaz ${ }^{4}$, \\ Jesús Carlos Ruvalcaba Ledezma ${ }^{5 *}$
}

${ }^{1}$ Master in public health, Autonomous University of the State of Hidalgo, Professor undergraduate oral public health and epidemiology, University Center Metropolitan Hidalgo.

${ }^{2}$ Students of the degree in dental surgeon in (CEUMH) University Center Metropolitan Hidalgo, Mexico.

${ }^{3}$ Research Professor full-time, academic Area of dentistry, Dept. of public health (UAEH).

Universidad Autónoma del Estado de Hidalgo, Mexico.

${ }^{4}$ Surgeon dentist (UdeG), University of Guadalajara, Mexico.

${ }^{5 *}$ Research full Professor, academic medicine, Department of public health (UAEH) Area. Autonomous University of the State of Hidalgo, Mexico.

Article No.: 121817178

DOI: 10.15580/GJEPH.2018.1.121817178

Submitted: 18/12/2017

Accepted: 28/12/2017

Published: 01/16/2018

${ }^{*}$ Corresponding Author

Jesús Carlos Ruvalcaba Ledezma

E-mail: dcspjcarlos@gmail.com

\section{Keywords:}

dental plaque, technical, oral hygiene brushing, thread dental, DMFT, oral health and diabetes mellitus.
Objective. To determine the CPO-D indices / IHOS in patient with Diabetes Mellitus of Tulancingo Hidalgo, Mexico.

Material and Methods. A descriptive observational transverse character study was conducted for 10 people of both sexes and aged 30/60 years with a diagnosis of $\mathrm{DM}$, the determination of the plaque plate is performed by means of revealing tablets to calculate the IHOS (index simplified Oral Hygiene) and the CPO-D index was determined by means of descriptive observation, by means of the method of clinical examination.

Results. Of the 10 patients examined, $100 \%$ represented dental plaque and cavities, not with the same index relationship existing between two factors such as; visit to the dentist, poor dental hygiene, poor brushing technique and null or inappropriate use of thread dental and mouth rinse. 


\section{INTRODUCTION}

The present study seeks to measure the presence of CPO-D/IHOS index and its relationship with poor oral hygiene of patients with Diabetes Mellitus between 30/60 years, belonging to Tulancingo Hidalgo. Given that oral diseases, are a major problem of public health in Mexico and with degrees more severe in patients with Diabetes Mellitus, we believe it is relevant information generated in this study (Sánchez, 2015; Gonzalez, 2015).

Diabetes Mellitus is a metabolic disease that occurs when the pancreas cannot produce enough insulin or when it fails to act on the body by which the cells do not respond to the stimulus (Carton, 2013; Valencia, 2014). This is a chronic disease in which the body cannot regulate the amount of glucose in the blood. Insulin is a hormone produced in the pancreas that allows food glucose to enter the cells of the body, where it becomes the energy needed to work the muscles and tissues (Benites, 2013). A person with diabetes does not properly absorb glucose and glucose is still circulating in the blood (a condition known as hyperglycemia), which damages the tissues of the body over time. This damage can lead to a disability and health complications that can be fatal (Coronel 2012; Juárez, 2012).

Diabetes mellitus is a syndrome characterized by the alteration in the metabolism of carbohydrates, fats and proteins, either by lack of insulin secretion, by decrease in the sensitivity of tissues to this hormone. Chronic hyperglycemia causes tissue damage. If blood sugar is not controlled well for a long period, blood vessels in many tissues of the body will begin to change and undergo structural changes, with the consequent deterioration of the supply of blood to the tissues, characteristic of the diabetes mellitus (Guyton 2001). In turn, this increases the risk of heart attack of myocardium, stroke, end-stage renal failure, retinopathy and blindness, as well as ischemia and gangrene (necrosis) of the extremities. Chronic hyperglycemia also harms many other tissues. For example, peripheral neuropathy (peripheral nerve function disorder) and alterations of the autonomic nervous system are frequent complications of diabetes mellitus chronic uncontrolled.

These alterations cause a reflex cardiovascular disorders, disorders of bladder control, decreased the sensitivity of the members and other symptoms of peripheral nerve injury. The exact mechanisms that induce tissue lesions from diabetes are not known completely, but they may likely be due to the numerous effects of hyperglycemia and other metabolic abnormalities on the proteins of endothelial cells and the vascular smooth muscle, as well as other tissues. In addition, patients with diabetes often develop a hypertension secondary to renal injury and atherosclerosis, secondary to abnormal metabolism of lipids that multiply the tissue damage caused by hyperglycemia (Suarez 2009; Benites 2013; Guyton, 2013).
As was mentioned in the skin, it is no exception that cutaneous manifestations of diabetes mellitus are numerous and varied, if consideration is given to the metabolic effects on the microcirculation and the changes in the collagen of the skin, the number of affected would increase (Valencia, 2014).

In the presence of hyperglycemia insulin deficit glucose will continue developing not insulin, this causes hypoxia, and alterations in the quality of the basal membranes (Valencia, 2014). Thickening in the capillaries of the whole organism occurs with most of hydroxyl sine and units of disaccharides and polysaccharides. Related to all the above is produced a series of alterations in microcirculation with increase in blood viscosity, circulation and permeability (Coronel 2012 ; Benites, 2013).

There is an imbalance between thromboxane and prostatic-clinics, increased Fibrinogen, factor inhibitor Fibrinogen and other clotting factors activator, which causes increased thrombogenesis. In the nonenzymatic glycosylation (GNE), when glucose binds to the amino groups of proteins of long life (e.g. collagen), it forms products terminals such as the 2-(2-furoyl)-4(5)-(2furanyl)-1H-imidazole, which is a compound of yellow fluorescent that gives a special hue to the skin. This reaction is irreversible and receives the name of late (Guyton 2001 Maillard reaction; Gonzalez, 2009).

Other terminal product, amino acid pentosidine, has been linked to the severity of the complications in diabetic patients. The cross-linking of collagen produced by the GNE gives it special characteristics such as: hardness is increased, its solubility decreases and there is increase of the resistance to enzymatic digestion. Many of the cutaneous manifestations in people with diabetes are related to these biochemical alterations (Coronel 2012; Juarez, 2013).

\section{The clinical manifestations can be divided into: \\ - Dermatosis in which diabetes acts as a determining factor. \\ - $\quad$ Skin infections. \\ - $\quad$ Other frequent associations. \\ - $\quad$ Changes caused by the treatment.}

People with diabetes are at the risk of developing a number of health problems that can result in disability or death. Constant high blood glucose levels can lead to serious diseases that affect the heart and blood vessels, eyes, kidneys and nerves. People with diabetes also have an increased risk of developing infections.

Diabetes is a threat to oral health. For example, there is an increased risk of inflammation of the gums (gingivitis) in people with poor glucose control. Gingivitis, in turn, is a major cause of tooth loss and can also increase the risk of cardiovascular disease.

Different studies have assessed the potential impact of diabetes in periodontal tissue. Most of them show that chronic hyperglycemia can significantly alter 
the health of this territory involving the physiology of this tissue at different levels.

The loss of periodontal attachment seems to be closely linked to the metabolic control of diabetes. The poor metabolic control is an important factor that can negatively modulate the evolution of periodontal disease. (Smith et Al., 2012).

Costa (2017) evaluated the influence of the periodontal status on changes in levels of hemoglobin glycosylated ( $\mathrm{HbA} 1 \mathrm{c})$ in patients with DM type 2 (T2dm) and concluded that the progression of periodontitis is associated with an increase of $\mathrm{HbA} 1 \mathrm{c}$ in patients with T2dm. Periodontitis may also be associated with an increase in the risk of diabetic complications, as observed in a longitudinal study of cases and controls in which $82 \%$ of diabetic patients with severe periodontitis experimentation emerged with cardiovascular events, stroke or vascular peripheral higher than $21 \%$ of the diabetic subjects (Mealey and Oates, 2006).

Tooth decay is a common disorder, which is followed in frequency by the common cold. It usually appears in children and young adults, but it can affect anyone. It is a common cause of loss of teeth in younger people.

The bacteria normally found in the mouth. These bacteria make food, especially sugars and starches, acids. Bacteria, acid, pieces of food and saliva combine in the mouth to form a sticky substance called plaque that adheres to the teeth. It is more common in molars, just above the line of the gums around the teeth and at the edges of fillings (Coronel 2012; Juarez, 2013).

The plaque, if not removed from the teeth becomes a substance called Tartar or calculus. Plaque and Tartar irritate the gums, causing gingivitis and periodontitis. The plaque starts to accumulate on the teeth within 20 minutes after eating. If this is not removed through conventional or clinical methods, starts bacterial proliferation and therefore all implications of this contract.

Plaque acids damage the enamel that covers the teeth causing demineralization and cavities in the tooth surfaces. Tooth decay usually does not hurt, unless they become very large and affect the nerves or cause a tooth fracture. Untreated, they can lead to a dental abscess. Tooth decay that is not treated also destroys the interior of the tooth (pulp), which requires a more extensive treatment, in the worst cases, the extraction of the tooth.

Carbohydrates (sugars and starches) increase the risk of tooth decay. The plaque plate is a mass formed by the growth and colonization of microorganisms on the surface of the teeth, restorations and dentures. (Coronel 2012; Juarez, 2013).

As these microorganisms adhere and grow, producing destructive substances for dental tissues (lactic acid), the plate is a complex microbial community; as a result of interactions between species, an ecological niche that favors the growth and survival of species occurs with strict anaerobic proteolytic, as well as appropriate conditions for the development of periodontitis (Juarez 2007 ; Vizcaya 2007). This is the result of a series of processes that involves a variety of bacteria, and components of the oral cavity. These processes include the formation of the film acquired on the surface of the dental organ. The colonization of microorganisms comprises several phases involving the deposition, adhesion, congregation, growth and production of microorganisms adhered on the acquired film.

Plate plaque is therefore the inefficiency of oral hygiene; improper use dental floss, mouthwash or even bad brushing techniques, make the amount of plate even more.

GENERAL OBJETIVE. To determine and compare the quality of oral health in the patients through preset indexes as well as relate their oral health with the general health condition in patients with controlled and non-controlled diabetes mellitus, and the quality of your oral health.

\section{SPECIFIC OBJECTIVES}

- Compare the seriousness of his State of health in controlled and uncontrolled patients.

- Determine the DMFT and risk indices to meet the oral health of the patients.

\section{METHOD}

\section{Participants}

This study is descriptive, observational transverse in character. Participation of in habitants with Diabetes Mellitus of both sexes, between 30 and 60 years, belonging to the municipality of Tulancingo Hidalgo, Mexico, were on a voluntary basis and through their consent for the filling of their medical history and a questionnaire for mediating variables with economic partners, this was how the results were obtained.

\section{Instruments and evaluation categories}

For the fulfillment of its objectives, a survey was designed. Which contained 2 categories; the first was the identification of patients, which was met with the rules of confidentiality and the second category was on oral hygiene and eating habits, and descriptive disease reporting.

\section{RESULTS}

We studied 10 patients (100\%). The average age was $30-60$, being $3(30 \%)$ male, 7 female $(70 \%)$. 


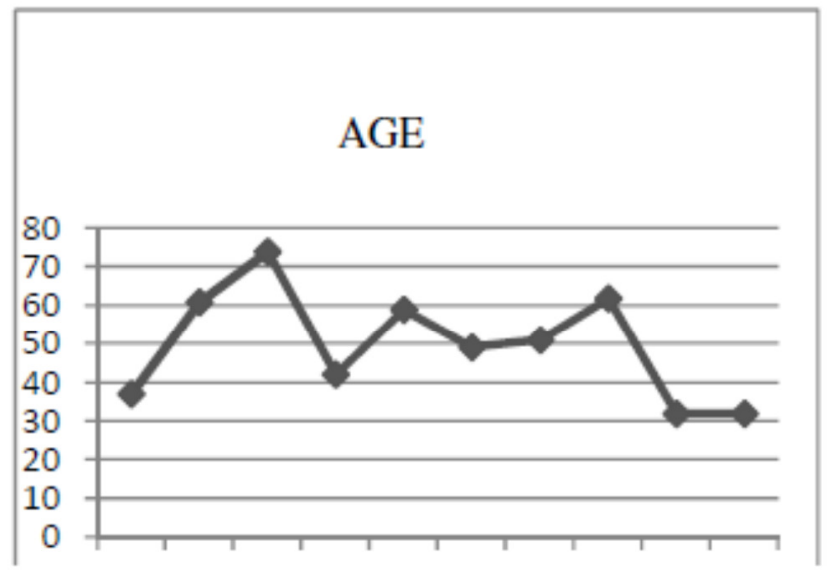

Figure 1 polygon frequency age of the patients. (April 2016)

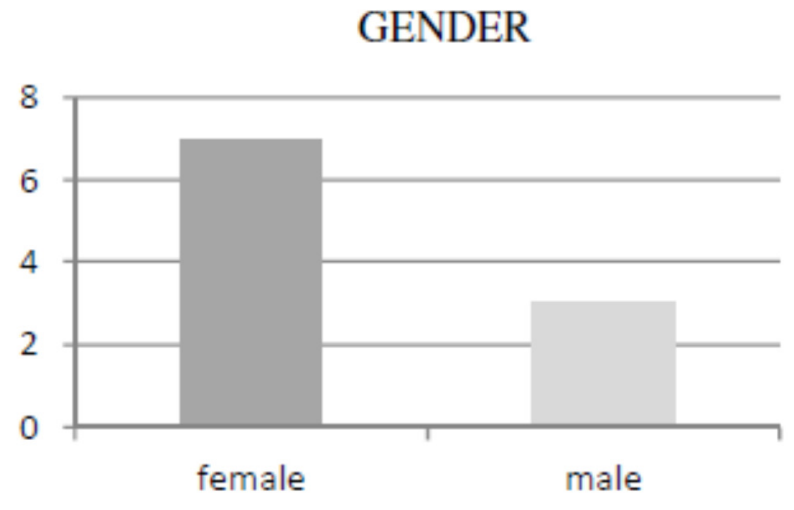

Figure 2 bar sex of the patients chart (April 2016)

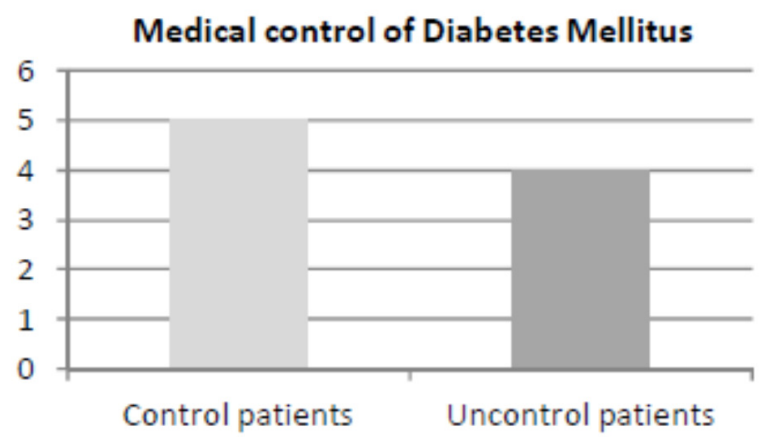

Figure 3 Bar of DM control (April 2016)

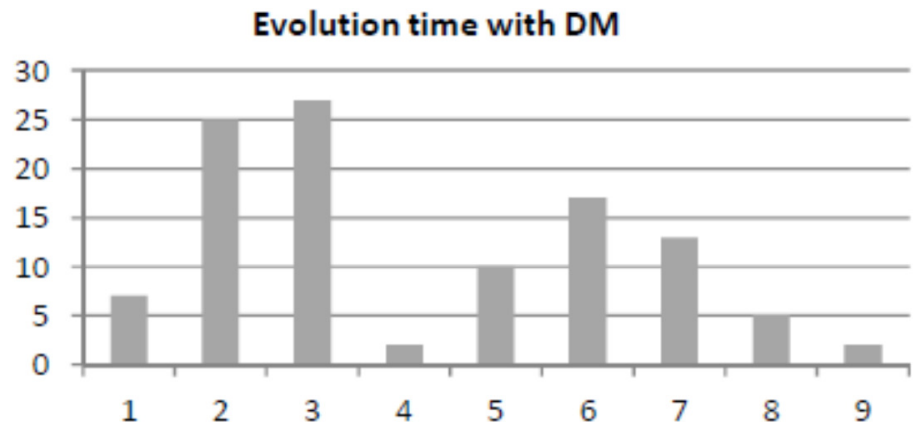

Figure 4 Bar of DM time evolution (April 2016)
Within the questionnaire were asked how many times a day patients brush their teeth, of which $40 \%$ answered that they brush 1 time a day, another $40 \%$ responded

\section{Brushing technique}

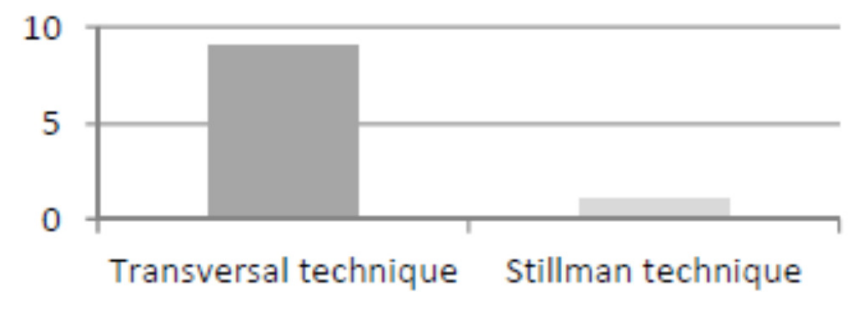

Figure 5 Bar brushing technique (April 2016)

Significantly, the surveys showed that $90 \%$ of the patients answered that they do not use dental floss and $10 \%$ use it. On the other hand, the results obtained that they brush 3 times or more a day. Taking into account that $90 \%$ use the cross brushing technique and just $10 \%$ using the Stillman technique.

\section{Brushing frequency}

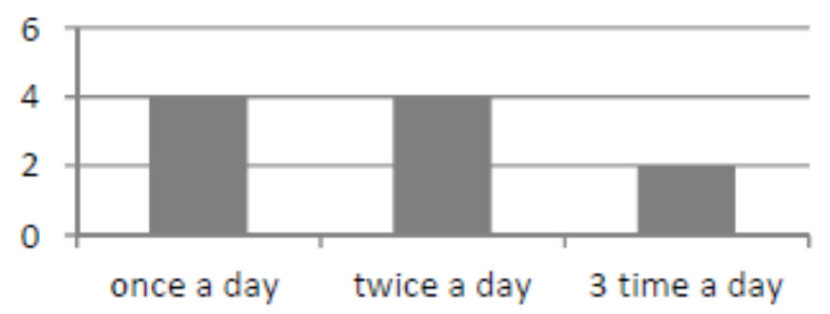

Figure 6 Bar brushing frequency (April 2016)

through the surveys reveal that the $56.25 \%$ rinse their mouths, while $43.75 \%$ do not rinse their mouths. 


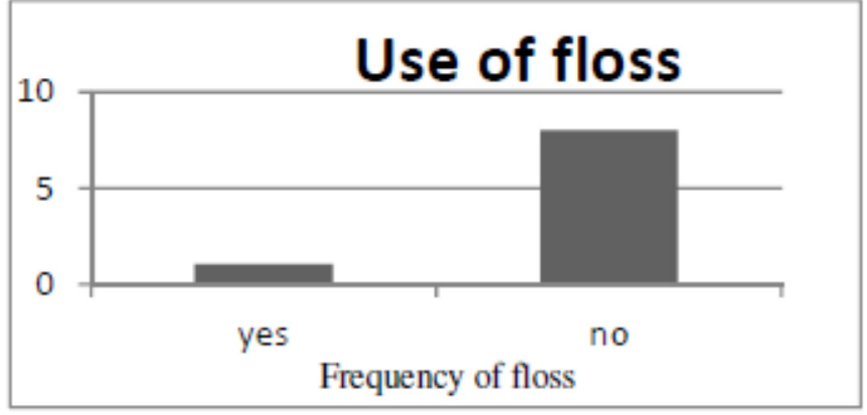

Figure 7 Bar of floss use (April 2016)

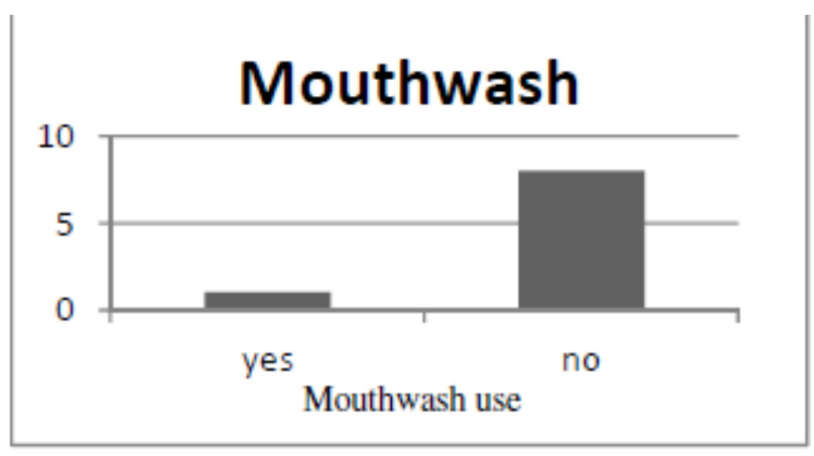

Figure 8 Bar of mouthwash use (April 2016)

Within the questionnaire, we asked patients if they were subjected to orthodontic treatment, $10 \%$ of the patients responded that they have orthodontic treatment, $90 \%$ of the surveyed patients responded not to have treatment of orthodontics. In the questionnaire applied to patients, it was important to ask them when was the last time they visited the dentist, as well as their last dental cleaning. $68.75 \%$ of the patients responded to less than 6 months,

$12.5 \%$ responded to more than 6 months and $18.75 \%$ had their last visit to the dentist to have been more than 1 year ago and in as far as the last dental cleaning only $10 \%$ was 8 months ago.

When performing the test with revealing plate pads, we failed to get the simplified oral hygiene index (risk), however, the analysis could find the following distribution.

Table 1. Index of simplified Oral Hygiene of patients with DM in Tulancingo Hidalgo, Mexico

\begin{tabular}{ccc}
\hline & \multicolumn{2}{c}{ IHOS } \\
\hline IHOS & Number of patients & Percentage \\
Bad & 6 & $60 \%$ \\
Regular & 3 & $30 \%$ \\
Good & 1 & $10 \%$ \\
\hline
\end{tabular}

To make the diagnosis of the DMFT index through the visual method - touch, we could find the following distribution.

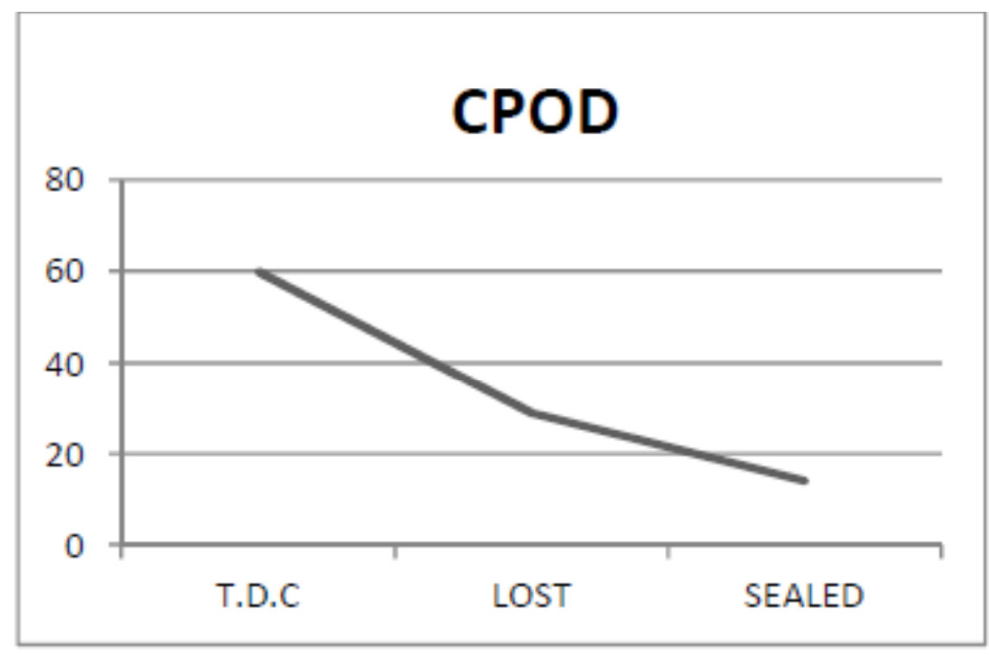

Figure 9 Bar of CPOD index of a patient population with DM in Tulancingo Hidalgo, Mexico (April 2016)

\section{DISCUSSION}

The DMFT index has higher prevalence in patients with Diabetes Mellitus, but especially with patients who do not use mouthwash flossing, do not brush teeth at least 3 times a day, do not have a good brushing technique and not visit the odontology frequently. It is of great 
importance to oral health, having good hygiene therefore, is learning the correct brushing techniques.

Stillman modified technique: consists of placing the bristles at $45^{\circ}$ with respect to the root of the teeth on the area of the gum, in a similar position to the Rotary method, resting partially on the gum. The brush is located horizontally and moving towards the occlusal (i.e. the edge of anterior teeth or chewing of the subsequent area), sweeping from the gingiva to the tooth. In this way the interproximal area (interdental) is cleaned and the gingival tissue is well handled.

Fones technique: the bristles of the brush are placed at $90^{\circ}$ to the long axis of the tooth and the brush is moved back and forward as it sweeps.
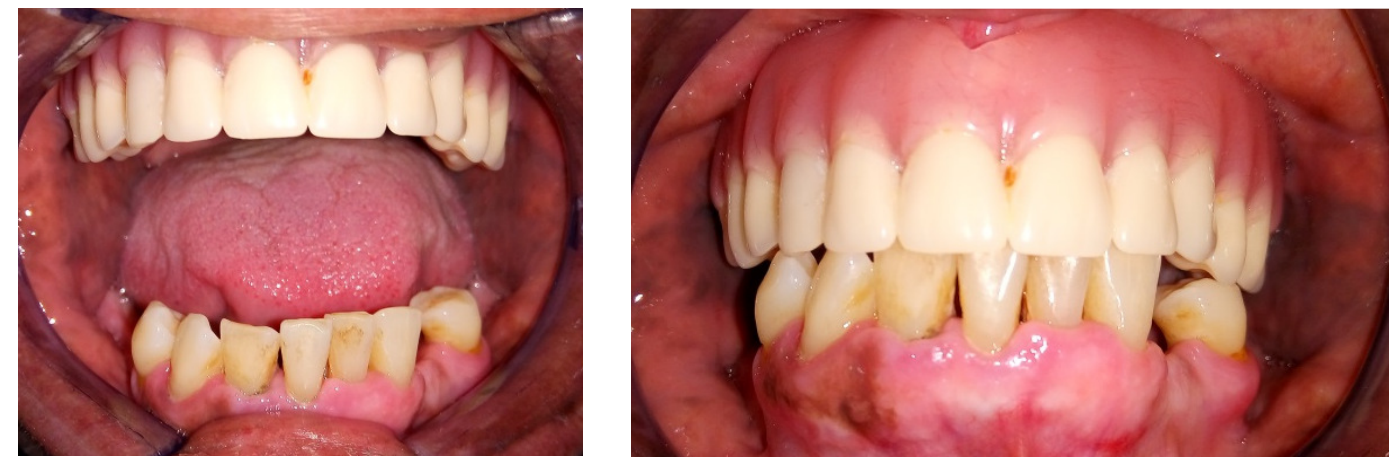

Patient 1. Female, in the pictures, we can see the severe deterioration of oral health, use of total superior prosthesis as well as the loss of several dental organs.
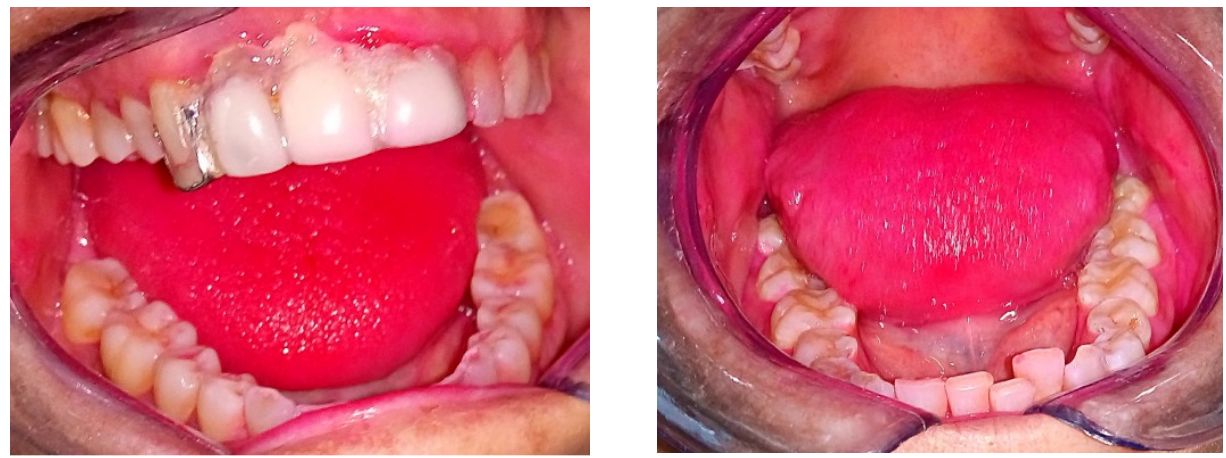

Patient 2. Women, in the photographs, we can observe the severe deterioration of oral health, use of completely mismatched partials as well as a high buildup of plaque plate in soft tissue and dental organs.
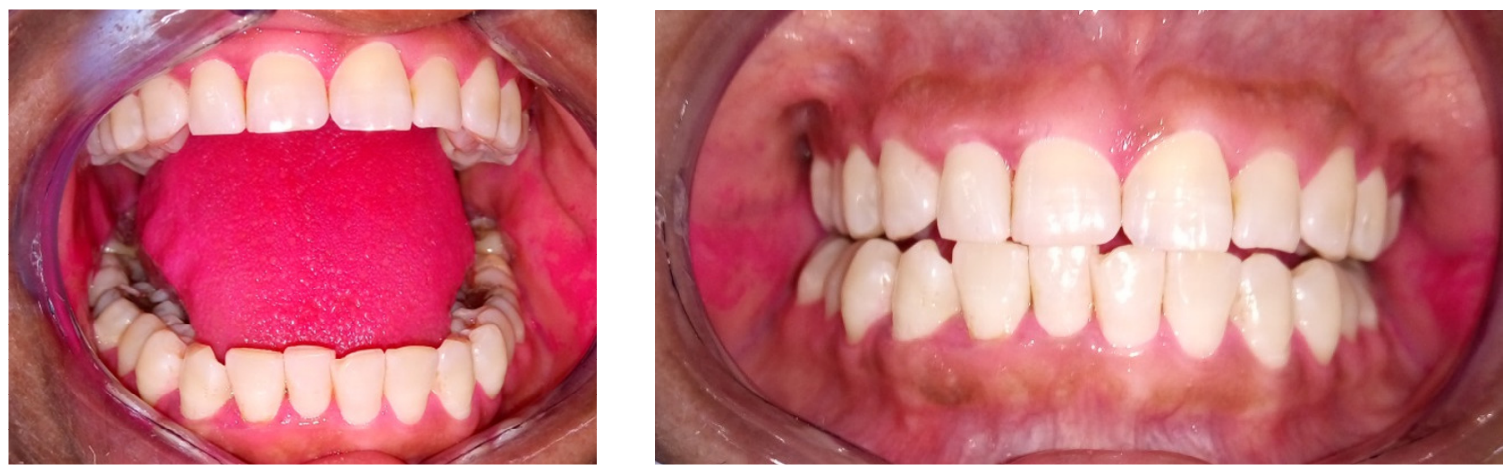

Patient 3. Women, in the photographs, we see moderate deterioration of oral health, mismatched seals and residual decay as well as a high accumulation of plaque plate in soft tissues. 

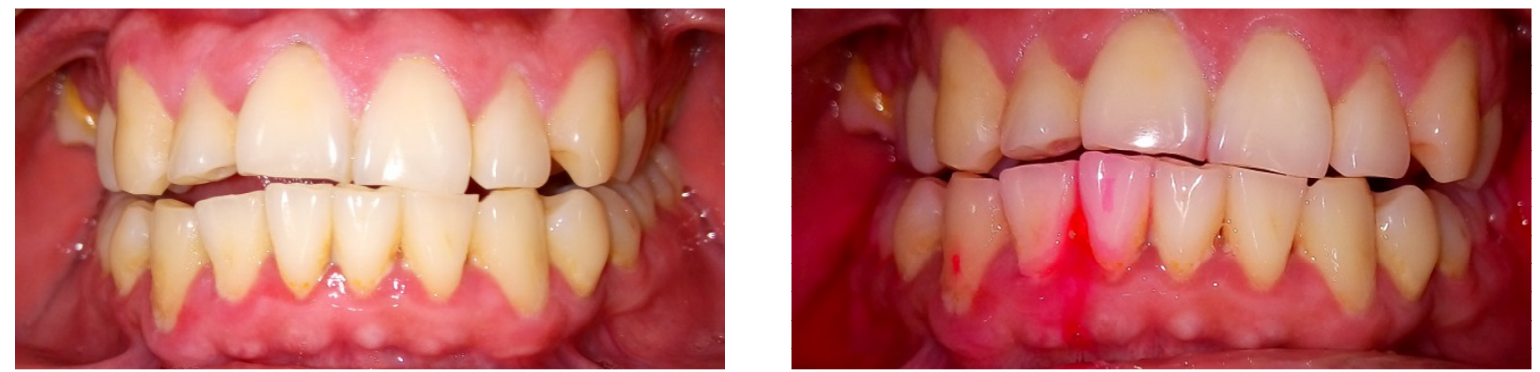

Patient 4. Masculine, in the photographs, we see moderate deterioration of the oral health, atypical wear dental bodies, edge-to-edge bite and a high buildup of plaque plate of specific areas, localized Gingivitis.
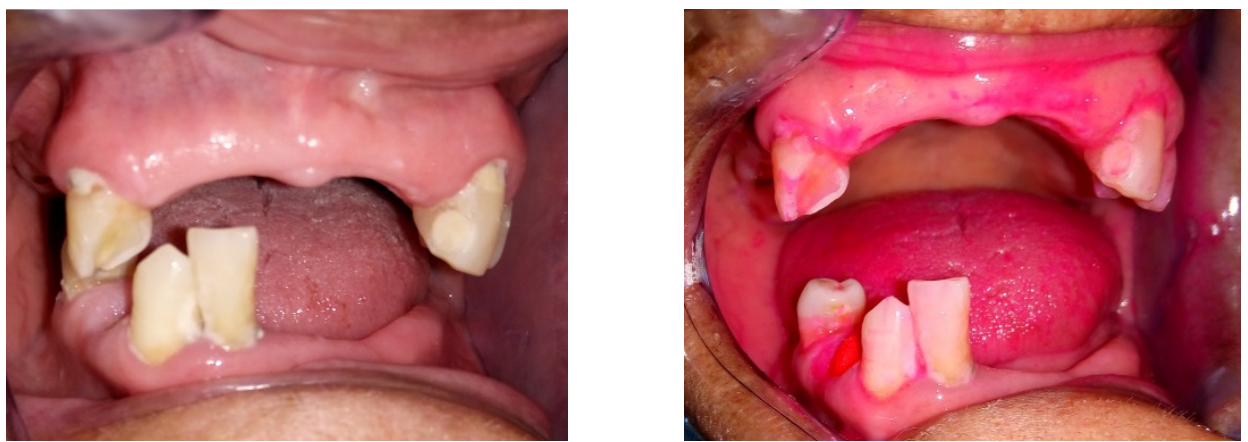

Patient 5. Female, in the photographs, we can observe the total deterioration of the oral health, atypical wear dental organs, great loss of dental bodies and high accumulation of plaque plate in soft tissue and dental remnants, patient matches the levels higher glucose in blood (patient not controlled).
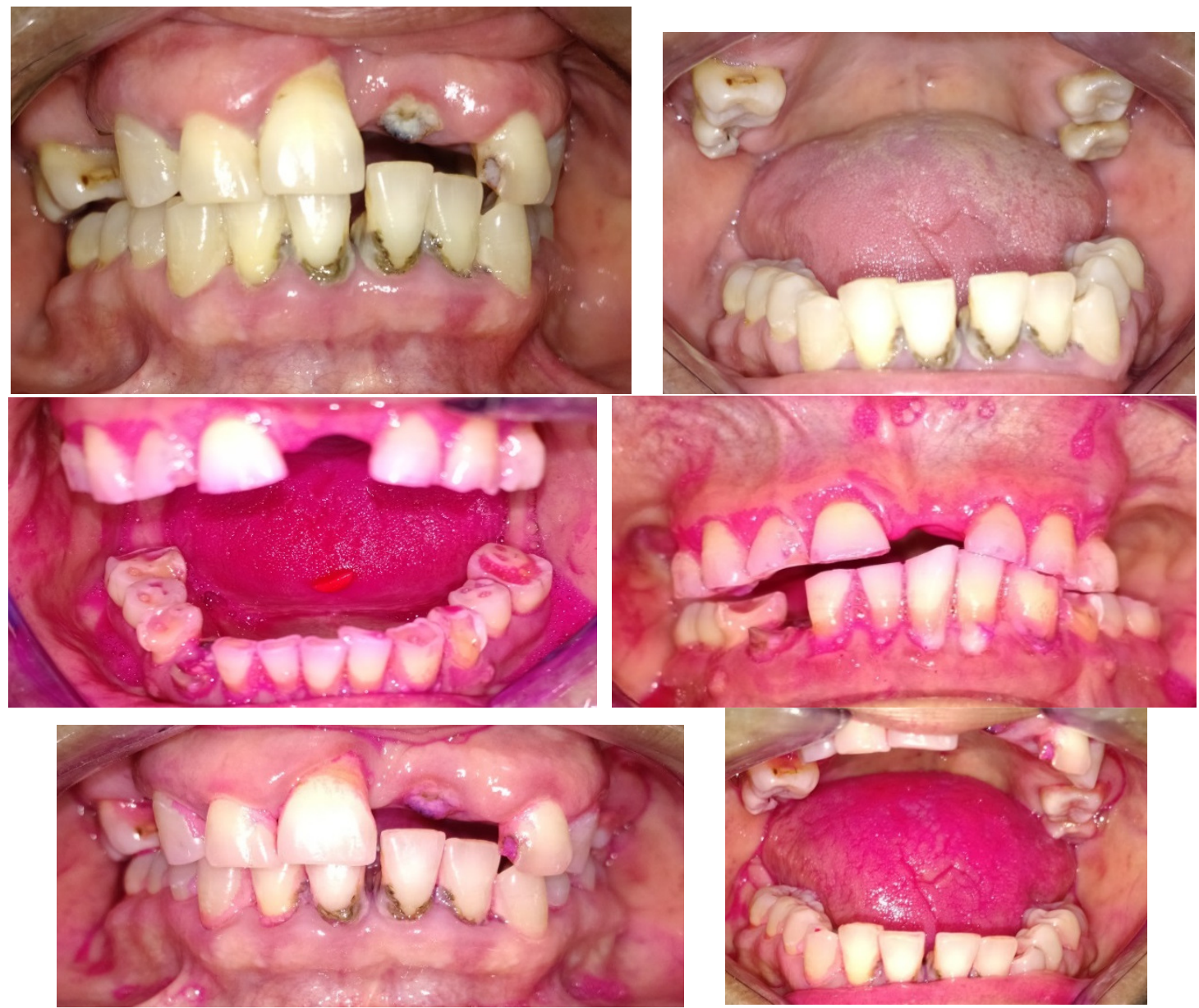

Patient 6. Masculine, the severe deterioration of the oral health, atypical wear dental organs, extrusion, excessive accumulation of dental Tartar, loss of dental organs, root fragments, and a high buildup of plaque plate can be seen on photographs. 


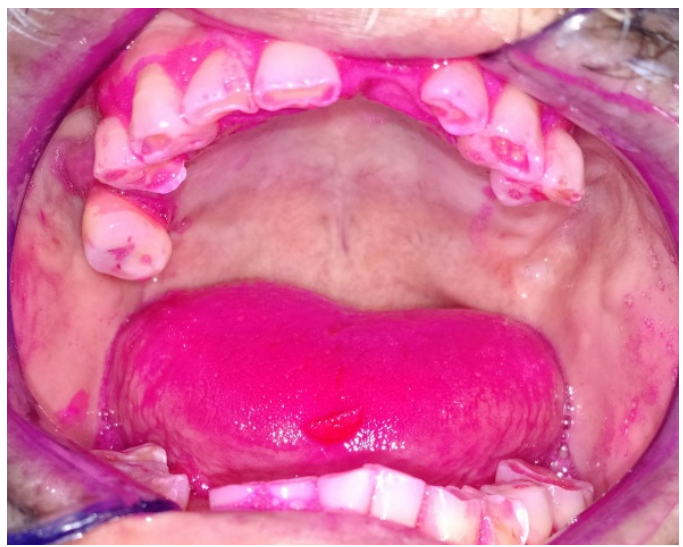

Patient 7. Masculine, in the photographs, we can observe the severe deterioration of the oral health, atypical wear dental organs (bruxism), extrusion, excessive accumulation of dental Tartar, loss of organs dental, root fragments, bite edge to edge and a high plate plaque buildup.

\section{CONCLUSIONS}

The prevalence of plate plaque and caries in patients was $100 \%$, all patients who performed the test presented plate plaque and caries; but not with the same index of plate. Patients with more plaque plate are those who do not attend often the dentist and never carried a dental cleaning appropriated by scarce resources.

Patients with a severe plaque index, have poor dental hygiene, do not use a good brushing technique and improperly rinse the mouth and do not know proper flossing. You can see how evolved health in diabetic patients is controlled and uncontrolled, especially as it is the process of degeneration of the dental organ and the way in which it has attacked significantly the uncontrolled diabetic patients.

\section{ACKNOWLEDGEMENTS}

The authors of the present research article would like to acknowledge and thank the collaboration of Yesenia Ruvalcaba Cobián Elizabeth who has a BA in Teaching as a Foreign Language Ingles, for her Contributions on the review and translation of the article; which allowed the possibility of transferring and modification of scientific knowledge.

\section{CONFLICT OF INTEREST}

The authors declare no conflict of interests for the publication of this research paper.

\section{REFERENCES}

Benites Sanchez, Byron Heman. (2013). periodontal disease in diabetic patients: systematic and physiological diseases. Republic of Ecuador. Edit. University of Guayaquil.

Brian L. Mealey and Thomas W. Oates. (2006). diabetes Mellitus and Periodontal Diseases. Journal of periodontology 1289-1303 (8) 77.

Colonel Tenorio, Carmen Yolanda. (2012). clinical and microbiological changes in periodontal treatment in diabetic patients with chronic adult periodontitis. Republic of Ecuador. Edit. University of Guayaquil.

Garcia, A. Alvares ML. (1998). Oral manifestations of diabetes mellitus. Venezuela. SciELO. Vol. 36 (2)

Gonzalez Figuero, RM. Jose Molina Lopez. (2009). oral microbiology. Fourth Edition publishers Mendez.

Guyton AC and Hall JE. (2001). treated Medical Physiology, McGraw-Hill Interamericana, $10^{\mathrm{a}}$ Edition.

James Carton. (2013). manual of clinical pathology. Mexico. Mc Graw Hill. Pp. 232.

Juarez, R. Mazzafero, see. Gorodner, JO. (2007). economic impact of hyperglycemia in type 2 diabetic patients oral health. Republic of Argentina.

Juarez, RP. Ricardo Chahin, j. (2012) knowledge, behaviors, perceptions and attitudes concerning oral health among diabetic patients. Republic of Argentina.

Katia L. Costa, Zuila A. Taboza, Gisele B. Los Angeles, Virginia R. Silveira, Renan Montenegro Jr., Alex N. Haas and Rodrigo O. Rego. (2017). Influence of periodontal disease on changes of glycated hemoglobin levels in patients whit type 2 diabetes mellitus: a retrospective cohort study. Journal of periodontology 88 (1), 17-25.

Margarita Vizcaya, M. Arduna, the. (2007). diabetes and health problems: there is a two-way relationship. Ciudad de la Habana Cuba. SciELO. ISSN 1561 $297 x$

Mejia Gonzalez, AM. Lomeli Buyoli, G. Marcela Cortés Gaxiola C. (2012). oral pathologies: standardized procedures manual. 
Pedro Francisco Valencia. (2014). pathology. Mexico DF. Mc Graw Hill. Pp.209.

Sanz-Sanchez I, Bascones Martínez A. (2008.22 September). Diabetes Mellitus: its implication in periodontal and oral pathology. Madrid Spain. Universidad Complutense de Madrid.

Smith, p., Retamal, i., Cáceres, M., Romero, A.M. Silva, D., Arancibia, r., \& Martinez, C. (2012). Diabetes and its impact on the periodontal territory. Clinical Journal of Periodontology, implantology and oral rehabilitation, 5 (2), 90-92.

Suarez Garcia, Ms. Ivernis Villega red, i. (2009). Prevention of periodontal disease in diabetic patients. Cuba. Journal of medical electronics-ISSN 1684-1824.

Cite this Article: Olvera PM, Alviter JAM, Fernández DLH, Javier M, Emmnuel LS, Alvarado PV, Diaz MLC, Ledezma JCR (2018). Analysis of indexes CPO-D and IHOS in patients with Diabetes Mellitus of Tulancingo Hidalgo, Mexico. Greener Journal of Epidemiology and Public Health, 6(1): 020-028, http://doi.org/10.15580/GJEPH.2018.1.121817178. 